\title{
Precision tools in hadron physics for Dalitz plot studies
}

\author{
Bastian Kubis ${ }^{* \dagger}$ \\ Helmholtz-Institut für Strahlen- und Kernphysik (Theorie) and \\ Bethe Center for Theoretical Physics, Universität Bonn, D-53115 Bonn, Germany \\ E-mail: kubis@hiskp.uni-bonn.de
}

Finding traces of new physics in Dalitz plot studies requires a precise understanding of the hadronic final-state interactions, which can be treated rigorously with methods from dispersion theory. I will show how these are currently applied and tested in various low-energy processes: setting limits on new-physics operators in lepton-flavor-violating $\tau$ decays; describing three-pion decays of vector mesons with high precision; and relating transition form factors to hadronic decays.

Xth Quark Confinement and the Hadron Spectrum,

October 8-12, 2012

TUM Campus Garching, Munich, Germany

\footnotetext{
* Speaker.

†Work supported in part by by DFG (SFB/TR 16), by DFG and NSFC (CRC 110), and by the project "Study of Strongly Interacting Matter" (HadronPhysics3, grant 283286) under the 7th Framework Program of the EU.
} 


\section{Dalitz plots and final-state interactions}

A precise study of final-state interactions is increasingly becoming of high importance for our understanding of diverse aspects of hadronic particle decays. They can be of significance for various reasons: if final-state interactions are strong, they can significantly enhance decay probabilities; they can significantly shape the decay probabilities, most prominently through the occurrence of resonances; besides resonances, also new and non-trivial analytic structures can occur, such as threshold or cusp effects [1]; and finally, they introduce strong phases or imaginary parts, the existence of which is e.g. a prerequisite for the extraction of CP-violating phases in weak decays. While strictly model-independent methods have been suggested to investigate CP violation in Dalitz plot analyses [2], requiring no hadron-physics input at all, it is obvious that the use of powerful methods such as analyticity, unitarity, and low-energy constraints from chiral symmetry will lead to a much more refined picture, and is probably the only way to investigate and identify the sources of new physics in precision studies, once it is found.

\section{Scattering and form factors}

Analyticity, unitarity, and crossing symmetry provide a high degree of constraint for the pionpion scattering amplitude. They can be exploited using dispersion relations, which can be formulated as a coupled system of partial-wave equations, the so-called Roy equations [3]. Modern precision analyses of the Roy equations have been performed [4], partly making use of constraints from chiral perturbation theory on the scattering lengths appearing as subtraction constants therein [5], and a similarly rigorous study exists also for pion-kaon scattering [6]. These provide us with highprecision parametrizations of the most relevant scattering amplitudes for light mesons appearing in the final states of heavy-meson decays.

Final-state interactions between only two strongly interacting particles as asymptotic states can be described in terms of form factors, which in turn can be linked to the properties of scattering amplitudes using analyticity and unitarity. As illustrated in Fig. 1, the unitarity relation for a form factor $F_{J}^{I}(s)$ (here: of the pion) of isospin $I$ and angular momentum $J$ reads

$$
\operatorname{disc} F_{J}^{I}(s)=2 i \operatorname{Im} F_{J}^{I}(s)=2 i F_{J}^{I}(s) \times \theta\left(s-4 M_{\pi}^{2}\right) \times \sin \delta_{J}^{I}(s) e^{-i \delta_{J}^{I}(s)},
$$

from which one immediately deduces Watson's final-state theorem [7]: the form factor shares the phase $\delta_{J}^{I}(s)$ of the (elastic) scattering amplitude. The solution to Eq. (2.1) is obtained in terms of

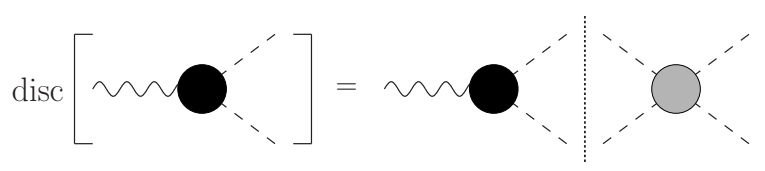

Figure 1: Graphical representation of the discontinuity relation for pion form factors. The black disc denotes the form factor, while the gray disc denotes the pion-pion scattering $T$-matrix, projected onto the appropriate partial wave. 
the Omnès function $\Omega_{J}^{I}(s)[8]$,

$$
F_{J}^{I}(s)=P_{J}^{I}(s) \Omega_{J}^{I}(s), \quad \Omega_{J}^{I}(s)=\exp \left\{\frac{s}{\pi} \int_{4 M_{\pi}^{2}}^{\infty} d s^{\prime} \frac{\delta_{J}^{I}\left(s^{\prime}\right)}{s^{\prime}\left(s^{\prime}-s\right)}\right\},
$$

where $P_{J}^{I}(s)$ is a polynomial. For the pion vector form factor, i.e., $J=1, I=1$, we assume a decrease of its modulus according to $1 / s$ for large $s$, as suggested by perturbative QCD, and that the phase $\delta_{1}^{1}(s)$ approaches $\pi$ in the same limit, which leads to a fall-off of $\Omega_{1}^{1}(s) \sim 1 / s$, such that the polynomial is required to be a constant. Gauge invariance finally requires the normalization to equal unity, such that $P_{1}^{1}(s) \equiv 1$. The representation (2.2) then holds to very good accuracy in the kinematic region where elastic unitarity is a reasonable approximation, which for the pion-pion $\mathrm{P}$-wave is phenomenologically found to be reliable up to about $1 \mathrm{GeV}$.

The description of the pion's scalar form factor(s), $J=0, I=0$, is slightly complicated by the strong impact of inelasticities generated by $K \bar{K}$ intermediate states right from the threshold $s=4 M_{K}^{2}$, which is enhanced due to the presence of the $f_{0}(980)$ resonance. The analogy to Eq. (2.1) is formulated as a matrix equation for the two-channel ( $\pi \pi$ and $K \bar{K}$ ) Muskhelishvili-Omnès problem, which, by an appropriate parametrization of the two-channel $T$-matrix, depends on three input functions, the $\pi \pi$ phase shift as well as modulus and phase of the $\pi \pi \rightarrow K \bar{K}$ amplitude. With similar assumptions on the asymptotics of form factor(s) and phases as before, the solution then depends on the normalizations of the scalar form factors of both pion and kaon at $s=0$, which, while not fixed exactly by gauge symmetry as in the case of the vector channel, can be very well constrained using chiral perturbation theory and lattice QCD simulations; see Ref. [9] and references therein for details. This holds for both scalar quark-antiquark source terms that can be considered, $(\bar{u} u+\bar{d} d) / 2$ and $\bar{s} s$, with obviously very different coupling strengths to pions and kaons.

An extremely important aspect of the discussion above is that the form factors characterizing the final-state interactions between two pions are universal, and may be applied in many different contexts in a model-independent way. In particular, they may be used to improve on the hadron physics aspects of new physics searches in low-energy precision studies. As an example, we discuss the lepton-flavor-violating decay $\tau \rightarrow \mu \pi^{+} \pi^{-}$[10]. It may be investigated based on an effective Lagrangian of the form

$$
\mathscr{L}_{\text {eff }}=\frac{\lambda_{V}}{2} \frac{1}{2}\left(\bar{u} \gamma^{\alpha} u-\bar{d} \gamma^{\alpha} d\right)\left(\bar{\mu} \gamma_{\alpha} \tau\right)+\left[\frac{\lambda_{S}^{n}}{2} \frac{1}{2}(\bar{u} u+\bar{d} d)+\frac{\lambda_{S}^{s}}{2} \bar{s} s\right](\bar{\mu} \tau)
$$

The effective current-current couplings $\lambda_{V}$ (vector-vector) and $\lambda_{S}^{n / s}$ (scalar-scalar) can be calculated from any underlying fundamental new-physics model; in Ref. [10], they have been derived from supersymmetric particle exchange with interactions given by an R-parity-violating superpotential. The matrix elements for the quark currents of Eq. (2.3) creating two pions out of the vacuum then precisely define the vector and scalar form factors discussed above.

In Fig. 2, we show the differential rates $d \Gamma\left(\tau \rightarrow \mu \pi^{+} \pi^{-}\right) / d s$, where $s$ is the invariant mass squared of the $\pi^{+} \pi^{-}$pair, assuming total dominance by one of the currents respectively, and setting the corresponding effective coupling arbitrarily to $1 \mathrm{GeV}^{-2}$. The bands in the scalar form factors are given by the uncertainty in the kaon form factor normalizations. We emphasize that both the spectral forms and the normalizations are constrained in a fully model-independent way: no assumptions on dominance by certain resonances (e.g., the $\rho^{0}(770)$ or the $f_{0}(980)$ ) or their specific 

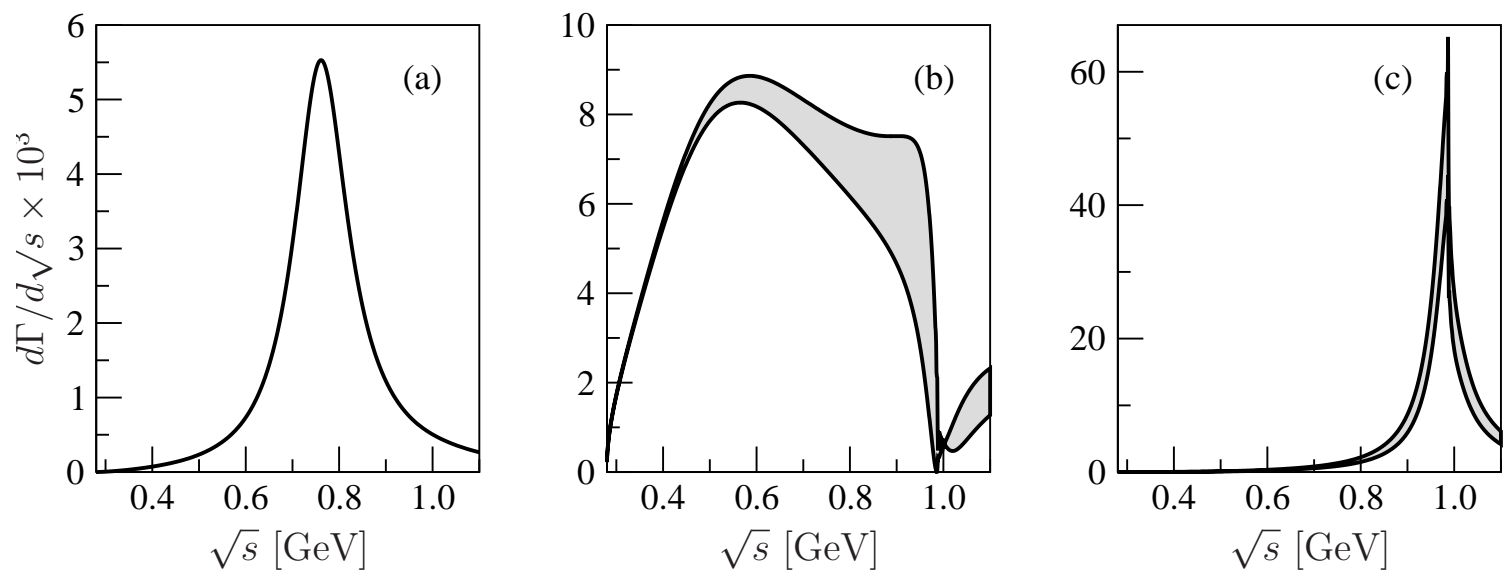

Figure 2: The predicted signals for $d \Gamma\left(\tau \rightarrow \mu \pi^{+} \pi^{-}\right) / d s$ for the currents (a) $\left(\bar{u} \gamma^{\alpha} u-\bar{d} \gamma^{\alpha} d\right) / 2$, (b) $(\bar{u} u+$ $\bar{d} d) / 2$, and (c) $\bar{s}$. In all cases the effective coupling constant is set to $1 \mathrm{GeV}^{-2}$. Figure taken from Ref. [10].

quark substructure need to be made, in contrast to alternative approaches [11]. Experimental upper limits on the decay $\tau^{-} \rightarrow \mu^{-} \pi^{+} \pi^{-}$have been given by the Belle collaboration [12] with and without kinematical cuts to isolate certain resonances. Integrating our theoretical spectra as shown in Fig. 2 in the appropriate invariant-mass ranges, we can translate these into limits on the products of fundamental coupling constants and supersymmetric masses, which prove significantly more restrictive than given in the literature so far [10].

\section{Dispersion relations for three-body decays}

The application of dispersion relations to three-body decays is more complicated than the treatment of form factors due to the more involved analytic structure, and the possibility of crossedchannel rescattering. Here, we concentrate on the three-pion decays of the lightest isoscalar vector mesons, $\omega / \phi \rightarrow \pi^{0} \pi^{+} \pi^{-}$[13]. We start by decomposing the amplitude $\mathscr{M}(s, t, u)$ according to

$$
\mathscr{M}(s, t, u)=i \varepsilon_{\mu v \alpha \beta} n^{\mu} p_{\pi^{+}}^{v} p_{\pi^{-}}^{\alpha} p_{\pi^{0}}^{\beta} \mathscr{F}(s, t, u),
$$

where $n^{\mu}$ is the polarization vector of the decaying $\omega / \phi$. Due to Bose symmetry, only partial waves of odd angular momentum contribute; neglecting discontinuities of F- and higher partial waves, $\mathscr{F}(s, t, u)$ can be further decomposed as $\mathscr{F}(s, t, u)=\mathscr{F}(s)+\mathscr{F}(t)+\mathscr{F}(u)$. The unitarity relation for $\mathscr{F}(s)$, assuming elastic final-state interactions, then leads to the following expression for the discontinuity of $\mathscr{F}(s)$ :

$$
\operatorname{disc} \mathscr{F}(s)=2 i\{\mathscr{F}(s)+\hat{\mathscr{F}}(s)\} \times \theta\left(s-4 M_{\pi}^{2}\right) \times \sin \delta_{1}^{1}(s) e^{-i \delta_{1}^{1}(s)},
$$

where $\delta_{1}^{1}(s)$ is the $\pi \pi \mathrm{P}$-wave phase shift. Were it not for the inhomogeneity $\hat{\mathscr{F}}(s)$, Eq. (3.2) would correspond to the discontinuity equation of the vector form factor, Eq. (2.1). The function $\hat{\mathscr{F}}(s)$ is 

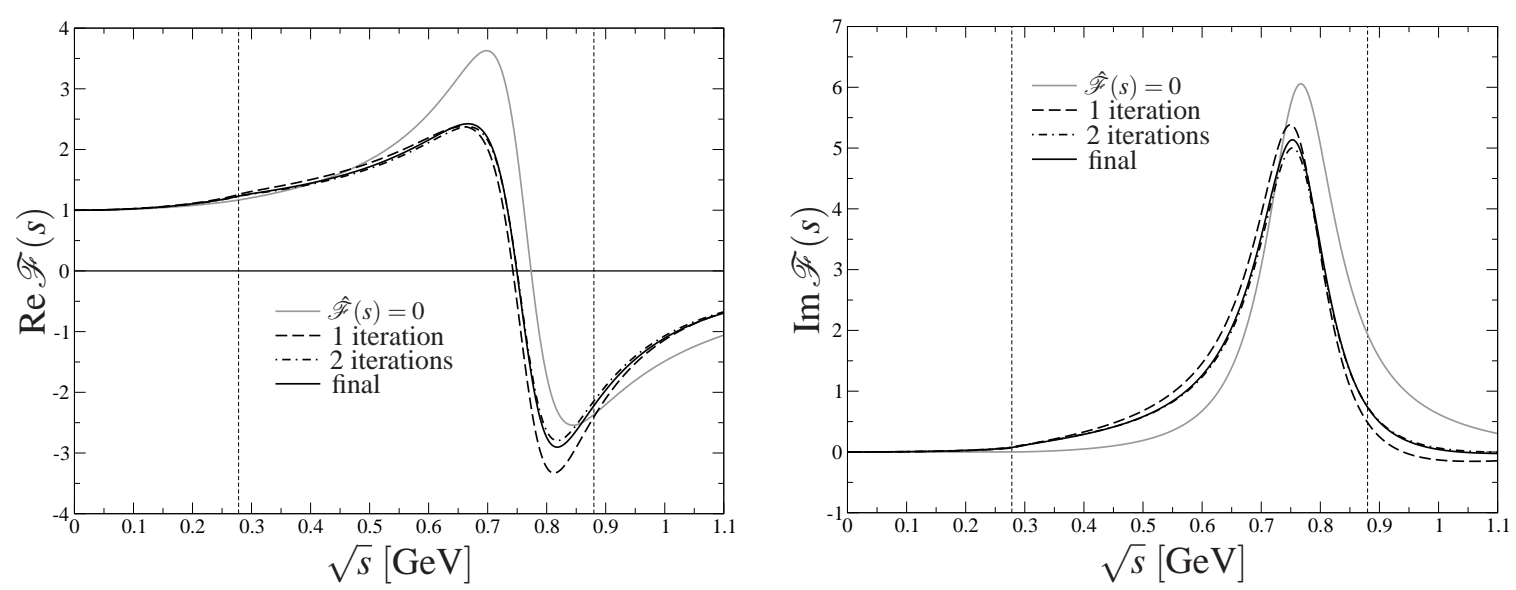

Figure 3: Successive iteration steps of real (left panel) and imaginary (right panel) part of the amplitude $\mathscr{F}(s)$ for $\phi \rightarrow 3 \pi$. The vertical dashed lines denote the physical region of the decay.

given by angular averages over $\mathscr{F}$ according to

$$
\begin{aligned}
\hat{\mathscr{F}}(s) & =3\left\langle\left(1-z^{2}\right) \mathscr{F}\right\rangle(s), & \left\langle z^{n} f\right\rangle(s) & =\frac{1}{2} \int_{-1}^{1} d z z^{n} f\left(\frac{1}{2}\left(3 s_{0}-s+z \kappa(s)\right)\right), \\
s_{0} & =\frac{M_{V}^{2}+3 M_{\pi}^{2}}{3}, & \kappa(s) & =\lambda^{1 / 2}\left(M_{V}^{2}, M_{\pi}^{2}, s\right) \sqrt{1-\frac{4 M_{\pi}^{2}}{s}}
\end{aligned}
$$

where $\lambda(x, y, z)=x^{2}+y^{2}+z^{2}-2(x y+x z+y z)$, and $M_{V}$ is the mass of the decaying vector meson. The angular integration including the $\kappa(s)$ function is non-trivial and generates a complex analytic structure, with three-particle cuts due to the fact that $\omega$ and $\phi$ are unstable and decay [13]. The analog to the Omnès solution (2.2) are then integral equations involving the inhomogeneity

$$
\mathscr{F}(s)=\Omega_{1}^{1}(s)\left\{a+\frac{s}{\pi} \int_{4 M_{\pi}^{2}}^{\infty} \frac{d s^{\prime}}{s^{\prime}} \frac{\sin \delta_{1}^{1}\left(s^{\prime}\right) \hat{\mathscr{F}}\left(s^{\prime}\right)}{\left|\Omega_{1}^{1}\left(s^{\prime}\right)\right|\left(s^{\prime}-s\right)}\right\},
$$

with the subtraction constant $a$. The number of subtractions is chosen such that the dispersion integral is guaranteed to converge.

Equations (3.3) and (3.4) can be solved iteratively: starting from an arbitrary input function $\mathscr{F}(s)$, we can calculate the inhomogeneity $\hat{\mathscr{F}}(s)$ according to Eq. (3.3), from which a new $\mathscr{F}(s)$ is obtained from Eq. (3.4); the procedure is stopped once a fixed point of the iteration is reached with sufficient accuracy. In the example discussed here, see Eq. (3.4), the subtraction constant works as an overall normalization factor of the solution; we match it to the partial decay width, but note that a normalized Dalitz plot distribution is subsequently a pure prediction. While the result is independent of the starting function, for the case at hand, we choose $\mathscr{F}(s)=a \Omega_{1}^{1}(s)$ in order to allow us to quantify crossed-channel effects (generated by the iteration) in a plausible way.

Figure 3 shows the result of such an iteration for the decay $\phi \rightarrow 3 \pi$ : it converges fast, with the third iteration already all but indistinguishable from the final result. The difference to the starting point of the iteration, the Omnès function without any crossed-channel rescattering, is, however, quite significant. The picture for $\omega \rightarrow 3 \pi$ (not shown here) is qualitatively similar, with convergence reached even faster (after two iterations, see Ref. [13]). 

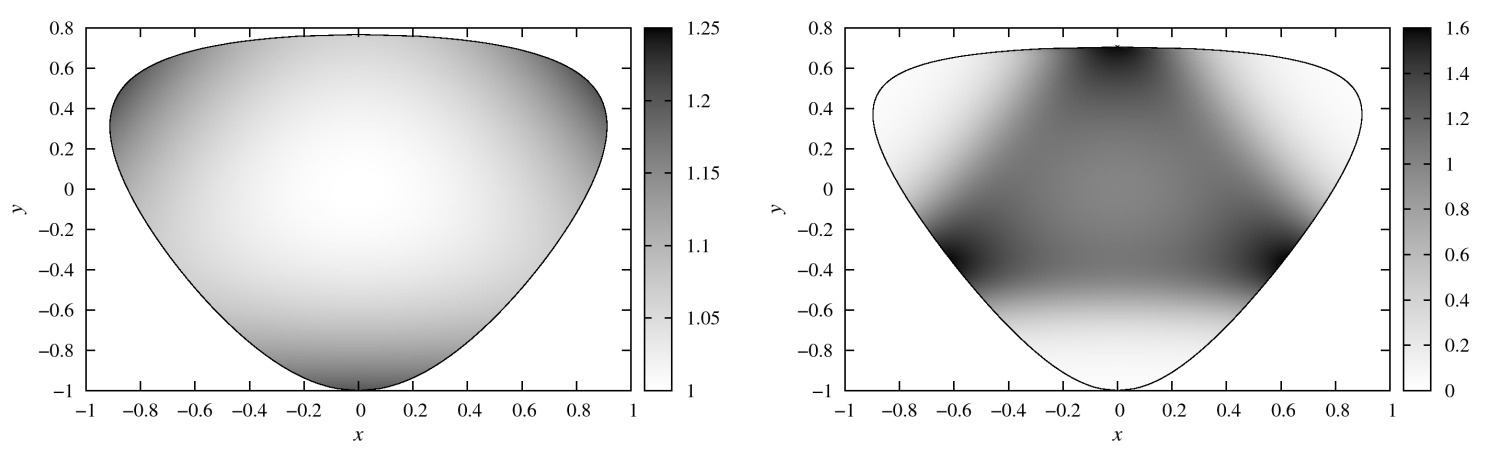

Figure 4: Dalitz plots for $\omega \rightarrow 3 \pi$ (left) and $\phi \rightarrow 3 \pi$ (right), normalized by the P-wave phase space.

The resulting Dalitz plots for both $\omega \rightarrow 3 \pi$ and $\phi \rightarrow 3 \pi$ are shown in Fig. 4, normalized by the P-wave phase space factor and using the kinematical variables

$$
x=\frac{t-u}{\sqrt{3} R_{V}}, \quad y=\frac{s_{0}-s}{R_{V}}, \quad R_{V}=\frac{2}{3} M_{V}\left(M_{V}-3 M_{\pi}\right) .
$$

Comparison to the experimental $\phi \rightarrow 3 \pi$ Dalitz plot of Ref. [14] shows that crossed-channel effects improve the reduced $\chi^{2}$ from $1.71 \ldots 2.06$ (with $\mathscr{F}(s)=a \Omega_{1}^{1}(s)$ ) to $1.17 \ldots 1.50$; further improvement and perfect agreement with the data can be achieved by introducing an additional subtraction constant in Eq. (3.4).

\section{Dispersion relations for transition form factors}

The $\omega / \phi \rightarrow \pi^{0} \gamma^{*}$ transition form factors, in addition to being interesting quantities in their own right, have attracted further attention due to their link to the doubly-virtual $\pi^{0}$ form factor $F_{\pi^{0} \gamma^{*} \gamma^{*}}\left(M_{\pi^{0}}^{2}, q_{1}^{2}, q_{2}^{2}\right)$ for fixed isoscalar photon virtualities, $q_{1}^{2}=M_{\omega}^{2}$. The latter, in turn, fixes the strength of the $\pi^{0}$ pole term in the hadronic light-by-light scattering contribution to the anomalous magnetic moment of the muon, which may soon constitute the dominant theoretical uncertainty in the determination of that quantity. See in particular Ref. [15] for an overview of the interrelations of the various form factors in this context, and Ref. [16] for an outline of how to utilize dispersive methods for an analysis of the $\pi^{0}$ form factor itself.

Assuming that the $\omega / \phi \rightarrow \pi^{0} \gamma^{*}$ transition form factor $f_{V \pi^{0}}(s)(V=\omega / \phi)$ is dominated by $\pi \pi$ intermediate states, one can derive the unitarity relation $[17,18]$

$$
\operatorname{disc} f_{V \pi^{0}}(s)=\frac{i s}{48 \pi} \sigma^{3}(s) f_{1}(s) F_{\pi}^{V *}(s), \quad \sigma(s)=\sqrt{1-\frac{4 M_{\pi}^{2}}{s}}
$$

where $f_{1}(s)=\mathscr{F}(s)+\hat{\mathscr{F}}(s)$ is the previously determined $V \rightarrow 3 \pi$ partial-wave amplitude and $F_{\pi}^{V}(s)=F_{1}^{1}(s)$ is the pion vector form factor. This relation leads to a once-subtracted dispersion relation

$$
f_{V \pi^{0}}(s)=f_{V \pi^{0}}(0)+\frac{s}{96 \pi^{2}} \int_{4 M_{\pi}^{2}}^{\infty} d s^{\prime} \frac{\sigma^{3}\left(s^{\prime}\right) f_{1}\left(s^{\prime}\right) F_{\pi}^{V *}\left(s^{\prime}\right)}{s^{\prime}-s},
$$

where the subtraction constant is fixed by the real-photon partial width $\Gamma_{V \rightarrow \pi^{0} \gamma}$. In principle the asymptotic behavior of the partial-wave amplitude and the pion vector form factor even allows 

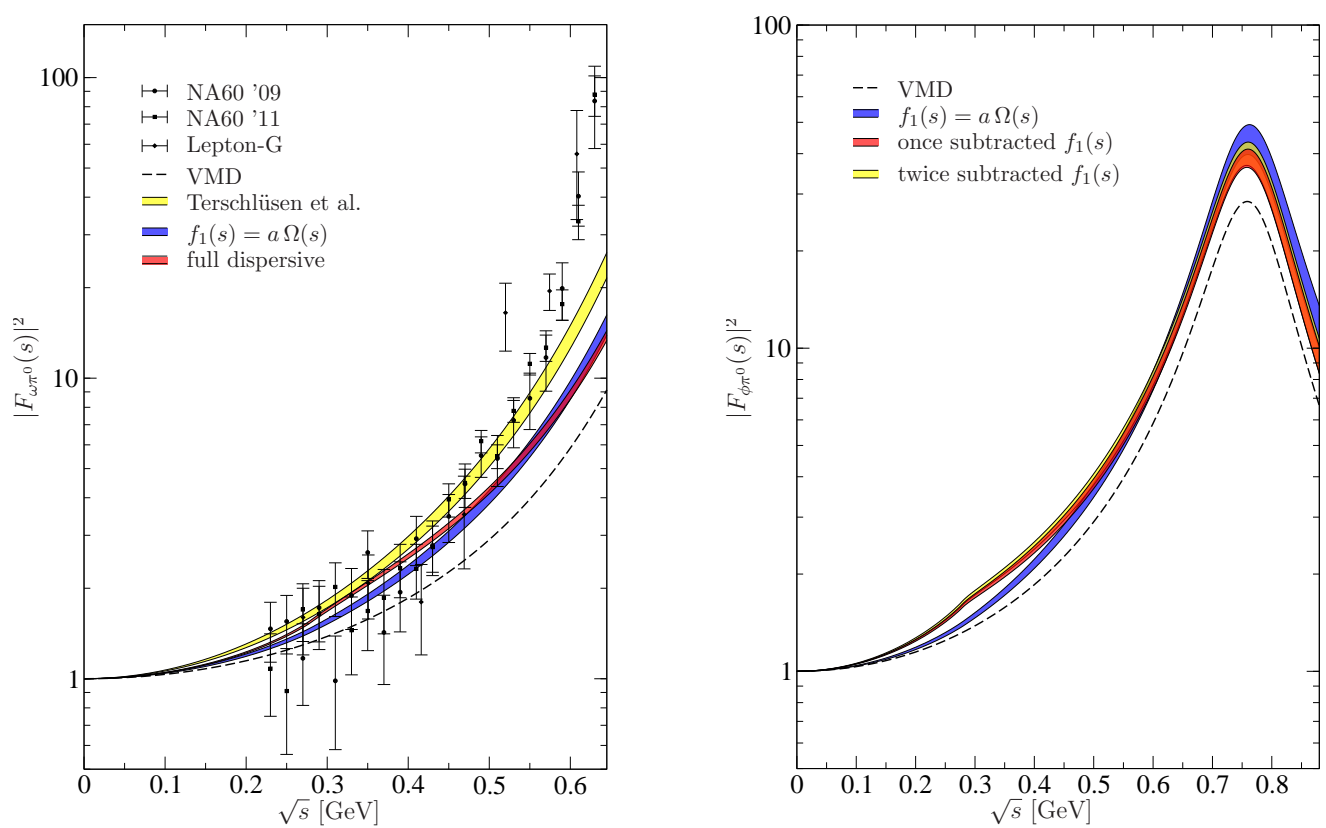

Figure 5: Left: normalized $\omega \rightarrow \pi^{0} \gamma^{*}$ form factor. We show pure VMD (with a finite width, dashed line), the results of a chiral Lagrangian treatment with explicit vector mesons [19] (yellow band), the dispersive solution for $f_{1}(s)=a \Omega(s)$ (blue band), and the full dispersive solution with one subtraction in the $V \rightarrow 3 \pi$ partial-wave amplitude (red band). The data is taken from Ref. [20]. Right: normalized $\phi \rightarrow \pi^{0} \gamma^{*}$ form factor. We compare pure VMD (dashed line), the dispersive solution for $f_{1}(s)=a \Omega(s)$ (blue band), and the full dispersive solution with one subtraction (red band) and two subtractions (yellow band).

for an unsubtracted dispersion relation. We have calculated $\Gamma_{V \rightarrow \pi^{0} \gamma}$ by a sum rule for $f_{V \pi^{0}}(0)$ and find that it is saturated to about $90-95 \%$ by two-pion intermediate states, thus justifying the approximation of neglecting inelastic effects.

In Fig. 5 we display the numerical results for the normalized transition form factor $F_{V \pi^{0}}(s)=$ $f_{V \pi^{0}}(s) / f_{V \pi^{0}}(0)$. Although we significantly improve on the vector-meson-dominance (VMD) result, we cannot reproduce the steep rise in the experimental data. Phenomenological monopole fits of the transition form factor lead to a pole close to the border of phase space of the decay, which cannot be accommodated within an approach that respects the strictures of analyticity and unitarity. We also find that three-particle effects in the partial-wave amplitude (compare the blue and red bands in the left panel of Fig. 5) do not perturb the spectrum in a way observable at the current precision level of the data.

We have to remark that our $\omega \rightarrow 3 \pi$ partial-wave amplitude is not yet backed up by experimental data; this caveat is absent in the case of the $\phi \rightarrow \pi^{0} \gamma^{*}$ transition form factor. The twicesubtracted partial-wave amplitude is an extremely precise representation of data, and thus all input in this channel is well constrained. Our numerical results again show enhancement over VMD, while crossed-channel rescattering effects are not particularly strong. We wish to point out that for this decay, the physical decay region encompasses the full energy range of the $\rho$ resonance; in contrast to $\omega \rightarrow \pi^{0} \gamma^{*}$ (or the OZI-favored $\phi \rightarrow \eta \gamma^{*}$ transition, where in the limit of isospin conservation the $\omega$ is the relevant resonance structure influencing the dilepton spectrum), any anomalous 
enhancement should therefore be directly accessible. We therefore strongly advocate an experimental investigation of $\phi \rightarrow \pi^{0} \ell^{+} \ell^{-}$in order to significantly advance our understanding of the vector-meson transition form factors.

Acknowledgments. I would like to thank the organizers of "Confinement X", and in particular those of the section on "QCD and New Physics", for the invitation to a most interesting and stimulating conference; and all my collaborators, who have shared their insights on the various topics discussed in these proceedings.

\section{References}

[1] J. Gasser, B. Kubis, A. Rusetsky, Nucl. Phys. B 850 (2011) 96 [arXiv:1103.4273 [hep-ph]].

[2] S. Gardner, Phys. Lett. B 553 (2003) 261 [arXiv:hep-ph/0203152]; S. Gardner, J. Tandean, Phys. Rev. D 69 (2004) 034011 [arXiv:hep-ph/0308228]; I. Bediaga, I. I. Bigi, A. Gomes, G. Guerrer, J. Miranda, A. C. dos Reis, Phys. Rev. D 80 (2009) 096006 [arXiv:0905.4233 [hep-ph]].

[3] S. M. Roy, Phys. Lett. B 36 (1971) 353.

[4] B. Ananthanarayan, G. Colangelo, J. Gasser, H. Leutwyler, Phys. Rept. 353 (2001) 207 [arXiv:hep-ph/0005297]; R. García-Martín, R. Kamiński, J. R. Peláez, J. Ruiz de Elvira, F. J. Ynduráin, Phys. Rev. D 83 (2011) 074004 [arXiv:1102.2183 [hep-ph]].

[5] G. Colangelo, J. Gasser, H. Leutwyler, Nucl. Phys. B 603 (2001) 125 [arXiv:hep-ph/0103088].

[6] P. Büttiker, S. Descotes-Genon, B. Moussallam, Eur. Phys. J. C 33 (2004) 409 [arXiv:hep-ph/0310283].

[7] K. M. Watson, Phys. Rev. 95 (1954) 228.

[8] R. Omnès, Nuovo Cim. 8 (1958) 316.

[9] M. Hoferichter, C. Ditsche, B. Kubis, U.-G. Meißner, JHEP 1206 (2012) 063 [arXiv:1204.6251 [hep-ph]].

[10] J. T. Daub, H. K. Dreiner, C. Hanhart, B. Kubis, U.-G. Meißner, arXiv:1212.4408 [hep-ph].

[11] M. J. Herrero, J. Portolés, A. M. Rodríguez-Sánchez, Phys. Rev. D 80 (2009) 015023 [arXiv:0903.5151 [hep-ph]].

[12] Y. Miyazaki et al. [Belle Collaboration], Phys. Lett. B 672 (2009) 317 [arXiv:0810.3519 [hep-ex]]; Phys. Lett. B 699 (2011) 251 [arXiv:1101.0755 [hep-ex]]; arXiv:1206.5595 [hep-ex].

[13] F. Niecknig, B. Kubis, S. P. Schneider, Eur. Phys. J. C 72 (2012) 2014 [arXiv:1203.2501 [hep-ph]].

[14] A. Aloisio et al. [KLOE Collaboration], Phys. Lett. B 561 (2003) 55 [Erratum-ibid. B 609 (2005) 449] [hep-ex/0303016].

[15] E. Czerwiński et al., arXiv:1207.6556 [hep-ph].

[16] M. Hoferichter, B. Kubis, D. Sakkas, Phys. Rev. D 86 (2012) 116009 [arXiv:1210.6793 [hep-ph]].

[17] G. Köpp, Phys. Rev. D 10 (1974) 932.

[18] S. P. Schneider, B. Kubis, F. Niecknig, Phys. Rev. D 86 (2012) 054013 [arXiv:1206.3098 [hep-ph]].

[19] C. Terschlüsen, S. Leupold, Phys. Lett. B 691 (2010) 191 [arXiv:1003.1030 [hep-ph]].

[20] R. I. Dzhelyadin et al., Phys. Lett. B 102 (1981) 296 [JETP Lett. 33 (1981) 228]; R. Arnaldi et al. [NA60 Collaboration], Phys. Lett. B 677 (2009) 260 [arXiv:0902.2547 [hep-ph]]; G. Usai [NA60 Collaboration], Nucl. Phys. A 855 (2011) 189. 